\title{
Como escolher entre as abordagens transolecraniana e por reflexão do tríceps para tratar fraturas distais do úmero em adultos: Um estudo prospectivo*
}

\section{How to Choose Between the Transolecranon and Triceps-Reflecting Approaches to Treat Distal Humerus Fractures in Adults: A Prospective Study}

\author{
Kalom Jamoh ${ }^{1}$ Gyneshwar Tonk ${ }^{2}$ \\ ${ }^{1}$ Departamento de Ortopedia, ESIC-PGIMSR, Nova Deli, Índia
${ }^{2}$ Departamento de Ortopedia, LLRM Medical College, Meerut, Uttar \\ Pradesh, Índia
}

Endereço para correspondência Kalom Jamoh, MS Orthopedic, Assistant Professor, Department of Orthopedics, ESIC-PGIMSR, Basaidarapur, New Delhi, India (e-mail: kalom19jamoh@gmail.com).

\section{Resumo \\ Palavras-chave \\ - fraturas do úmero \\ - processo olecraniano \\ - osteotomia \\ Objetivo Escolher uma abordagem posterior adequada para fraturas distais do úmero em adultos. \\ Métodos Cinquenta pacientes com fraturas distais do úmero foram analisados prospectivamente. As fraturas foram classificadas por meio da classificação Arbeitsge- meinschaft für Osteosynthesefragen/Orthopaedic Trauma Association (AO/OTA). Os pacientes foram divididos em grupo A e grupo B. A osteotomia olecraniana (aborda- gem transolecraniana) foi realizada em 30 pacientes, e a abordagem por reflexão do tríceps foi usada em 20 pacientes. Os resultados funcionais foram avaliados por meio do Mayo Elbow Performance Score (MEPS) e do questionário Disabilities of the Arm, Shoulder and Hand (DASH). \\ Resultados O tempo médio da cirurgia foi de $92,62 \pm 8,73$ minutos para o grupo A, e de $78,63 \pm 7,02$ minutos para o grupo $B(p<0,01)$ e a média da perda sanguínea foi de $222,78 \pm 34,93 \mathrm{~mL}$ no grupo $A$, e de $121,61 \pm 19,85 \mathrm{~mL}$ no grupo $B(p<0,01)$, os quais foram estatisticamente significativos. As pontuações médias no MEPS e no DASH de ambos os grupos foram consideradas insignificantes. Complicações como infecção, neurapraxia e irritação de tecidos moles foram mais observadas no grupo A.}

Estudo desenvolvido no Departamento de Ortopedia, ESIC-PGIMSR,

Nova Deli, Índia.

recebido

19 de Maio de 2021

aceito

20 de Setembro de 2021

Publicado on-line

Fevereiro 9, 2021
DOI https://doi.org/ $10.1055 / \mathrm{s}-0041-1741444$. ISSN 0102-3616. (c) 2021. Sociedade Brasileira de Ortopedia e Traumatologia. All rights reserved.

This is an open access article published by Thieme under the terms of the Creative Commons Attribution-NonDerivative-NonCommercial-License, permitting copying and reproduction so long as the original work is given appropriate credit. Contents may not be used for commercial purposes, or adapted, remixed, transformed or built upon. (https://creativecommons.org/ licenses/by-nc-nd/4.0/)

Thieme Revinter Publicações Ltda., Rua do Matoso 170, Rio de Janeiro, RJ, CEP 20270-135, Brazil 


\begin{abstract}
Keywords

- humeral fractures

- olecranon process

- osteotomy

Objective To choose an appropriate posterior approach for distal humerus fractures in adults.

Methods Fifty patients with distal humerus fractures were analyzed prospectively. The fractures were classified using the Arbeitsgemeinschaft für Osteosynthesefragen/ Orthopaedic Trauma Association (AO, Working Group for Bone Fusion Issues, in German/OTA) classification. The patients were divided into group A and group B. Olecranon osteotomy (the transolecranon approach) was performed in 30 patients, and the tricepsreflecting approach was used in 20 patients. The functional results were evaluated using the Mayo Elbow Performance Score (MEPS) and the Disabilities of the Arm, Shoulder and Hand (DASH) questionnaire.

Results The average operative time was of $92.62 \pm 8.73$ minutes for group $A$, and of $78.63 \pm 7.02$ minutes for group $B,(p<0.01)$, and the average blood loss was of $222.78 \pm 34.93 \mathrm{~mL}$ for group $A$, and of $121.61 \pm 19.85 \mathrm{~mL}$ for group $\mathrm{B},(p<0.01)$, which were statistically significant. The mean scores on the MEPS and DASH of both groups were found to be insignificant. Complications like infection, neurapraxia and soft tissue irritation where observed more in group A.

Conclusion The triceps-reflecting approach results in a shorter operative time, a lower levels of blood loss, and a low rate of complications, and olecranon osteotomy provides better accuracy in terms of articular reduction. But there were no significant differences between the two groups regarding the functional outcome. Therefore, we have proposed a new classification that is a modification of the AO/OTA classification: type 1 includes AO grades 13A to C2 (B3 excluded); and type 2, AO 13C3. For type-1 fractures, the triceps-reflecting approach may be considered, and, for type-2 fractures, olecranon osteotomy.
\end{abstract}

Conclusão A abordagem por reflexão do tríceps resulta em menor tempo de operação, menor perda de sangue, e baixas taxas de complicações, e a osteotomia olecraniana proporciona uma melhor precisão da redução articular. Mas não houve diferença significativa entre os dois grupos em termos do resultado funcional. Por isso, propusemos uma nova classificação, que é uma modificação da classificação AO/OTA: o tipo 1 inclui os graus AO 13A a C2 (excluído o B3); e o tipo 2, AO 13C3. Para fraturas do tipo 1 , a abordagem por reflexão do tríceps pode ser considerada, e, para as fraturas do tipo 2, a osteotomia olecraniana.

\section{Introdução}

As fraturas distais do úmero constituem aproximadamente $2 \%$ de todas as fraturas. ${ }^{1}$ Elas têm distribuição etária bimodal, com pico de incidência entre os 12 e 19 anos, geralmente em homens, e em mulheres com idade superior a 80 anos. Em adultos jovens, as fraturas são tipicamente causadas por lesões de alta energia, como por colisões automobilísticas, quedas de altura, atividade esportiva, acidentes industriais, e ferimentos por armas de fogo. ${ }^{2}$ Em idosos, as fraturas geralmente são causadas por lesões de baixa energia, como quedas ao solo.

As fraturas distais do úmero continuam sendo algumas das lesões mais desafiadoras de se tratar. Elas são comumente multifragmentadas, e têm anatomia complexa, com opções limitadas de fixação interna. ${ }^{2,3}$ Os desfechos do tratamento são frequentemente associados a rigidez do cotovelo, instabilidade e dor. Deseja-se uma articulação de cotovelo indolor, estável e móvel, pois isso permite que a mão realize as atividades da vida diária, principalmente a higiene pessoal e a alimentação. Portanto, começar com um úmero distal altamente traumatizado e terminar com uma articulação estável, móvel e livre de dor exige uma abordagem inteligente. A osteotomia olecraniana (também conhecida como abordagem transolecraniana) é a abordagem cirúrgica preferida na literatura, e proporciona excelente exposição. No entanto, ela tem suas desvantagens, como consolidação atrasada, não consolidação, ossificação heterotópica, fraqueza do mecanismo extensor e implante proeminente. Para evitar tais complicações, uma abordagem posterior do úmero distal, que preserve o mecanismo extensor e reflita o tríceps, por meio de uma incisão posterior da linha média foi sugerida por muitos cirurgiões. ${ }^{4}$ Realizamos este estudo para avaliar o desfecho de pacientes adultos com fraturas distais do úmero tratadas com as abordagens por reflexão do tríceps e por osteotomia olecraniana em termos 
de: precisão da redução articular, tempo de cirurgia, perda de sangue, resultados funcionais e complicações imediatas, precoces e tardias.

\section{Métodos}

Este estudo prospectivo foi realizado em um hospital após a liberação do comitê de ética (S-1/2019/9159). De dezembro de 2017 a março de 2021, os prontuários de 50 pacientes com idades entre 20 e 70 anos foram avaliados. Eles foram divididos em dois grupos: O grupo A continha os pacientes operados por osteotomia olecraniana, e o grupo $\mathrm{B}$, os pacientes operados por reflexão do tríceps. A randomização foi feita com base no número de internação do paciente (um número par foi submetido a osteotomia olecraniana, e um número ímpar, ao procedimento de reflexão do tríceps). Todas as fraturas fechadas, bem como as fraturas abertas do úmero distal de tipo 1 na classificação de Gustillo e Anderson foram incluídas em nosso estudo. Foram excluídas as fraturas expostas do úmero distal de tipos II e III, pacientes com menos de 20 anos ou mais de 70 anos, fraturas com lesões vasculares associadas, pacientes não cooperativos, lesões de mais de 3 semanas, todas as fraturas do úmero distal patológicas devido a patologia neoplásica ou infecciosa (ativa ou sequelas), e os pacientes politraumatizados.

Após o atendimento imediato de emergência, foi feita estabilização hemodinâmica do paciente e talas foram colocadas nos membros afetados. Todos os dados demográficos do paciente, juntamente com o número de contato e endereço, foram registrados. Após uma anamnese minuciosa, o paciente foi avaliado clinicamente pela primeira vez, e verificou-se se a lesão era fechada ou aberta, e a classificação de Gustilo e Anderson foi aplicada nas fraturas abertas. Foram feitos raios $\mathrm{X}$ do cotovelo afetado (nas vistas anteroposterior e lateral), e as fraturas foram classificadas de acordo com o sistema de classificação Arbeitsgemeinschaft für Osteosynthesefragen/ Orthopaedic Trauma Association (AO/OTA). No entanto, em caso de dúvida quanto à extensão intra-articular da fratura ou a sua classificação, foi feita uma tomografia computadorizada (TC) do cotovelo afetado, e essa avaliação foi feita principalmente nas fraturas do tipo $\mathrm{C} 2$ da AO/OTA.

\section{Procedimento Operatório}

O paciente foi posicionado em decúbito lateral com o membro afetado pendurado sobre um apoio, e foi aplicado torniquete no braço superior em ambos os procedimentos.

\section{Osteotomia olecraniana}

Uma incisão longitudinal de linha média foi feita no aspecto posterior do cotovelo, começando aproximadamente $7,5 \mathrm{~cm}$ a $10 \mathrm{~cm}$ acima do olécrano e estendendo-se de $5 \mathrm{~cm}$ a $7 \mathrm{~cm}$ distalmente a partir da ponta do processo olecraniano. Logo acima da ponta do olécrano a incisão foi curvada lateralmente para que não causasse necrose na ponta de rolamento de peso do olécrano e a afastasse dos dispositivos usados para corrigir a osteotomia. A incisão foi curvada medialmente novamente de modo que cobrisse o meio da superfície subcutânea da ulna. A fáscia profunda foi incisada na linha média. O nervo ulnar foi palpado no sulco ósseo atrás do epicôndilo medial, e a fáscia excessiva foi incisada para expor o nervo. O nervo ulnar foi dissecado completamente e envolto com um tubo de alimentação infantil para proteção. Uma osteotomia em forma de $\mathrm{V}$ com ápice dirigido distalmente foi feita a cerca de $2 \mathrm{~cm}$ da ponta do olécrano por perfuração múltipla com fio guia ou fio de Kirschner, seguida de osteotomia. $\mathrm{O}$ osso foi dividido até ser cortado quase inteiramente. Os anexos de tecido mole das faces medial e lateral do olécrano que haviam sido submetidos a osteotomia e retraídos proximalmente foram liberados, e o tríceps da parte de trás do úmero foi elevado e costurado à pele do braço superior. Uma dissecção subperiosteal em volta das bordas medial e lateral do osso permitiu a exposição de todas as superfícies do úmero distal, exceto a anterior. Todos os anexos de tecido mole ao osso que poderiam ser preservados foram retidos, particularmente durante a redução da fratura. O nervo ulnar foi mantido afastado do campo operacional durante todas as etapas da dissecação. A superfície articular do úmero distal e ambos os pilares foram restaurados, a fixação foi feita por placas e parafusos, e a amplitude de movimento (ADM)foi verificada na mesa de operação. 0 nervo ulnar foi removido de seu lugar e transposto anteriormente para evitar o impacto do implante. O reparo da osteotomia foi feito com fios de banda de tensão, com dois fios de Kirschner (fios $\mathrm{K}$ ) e fio de aço inoxidável (fio $\mathrm{AI}$ ) (-Figuras 1-4).

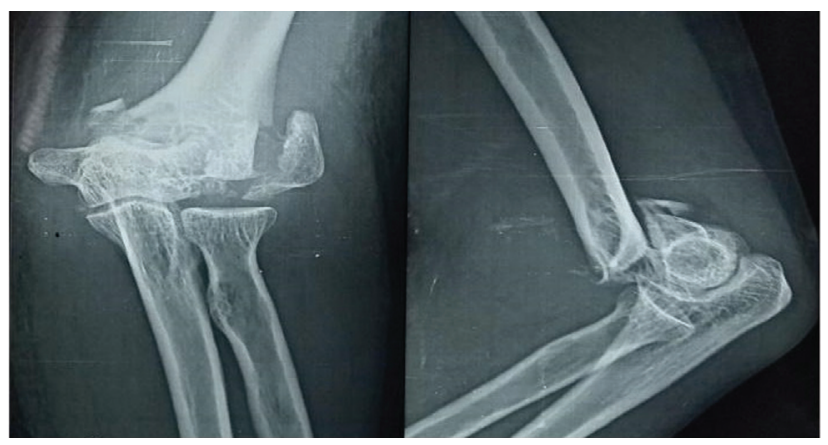

Fig. 1 Abordagem transolecraniana do caso-1: raio X pré-operatório.

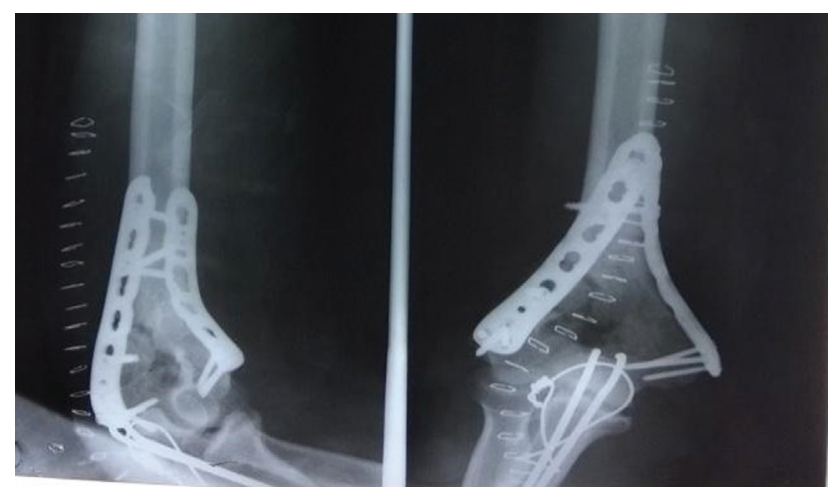

Fig. 2 Abordagem transolecraniana do caso-1: raio X pós-operatório. 


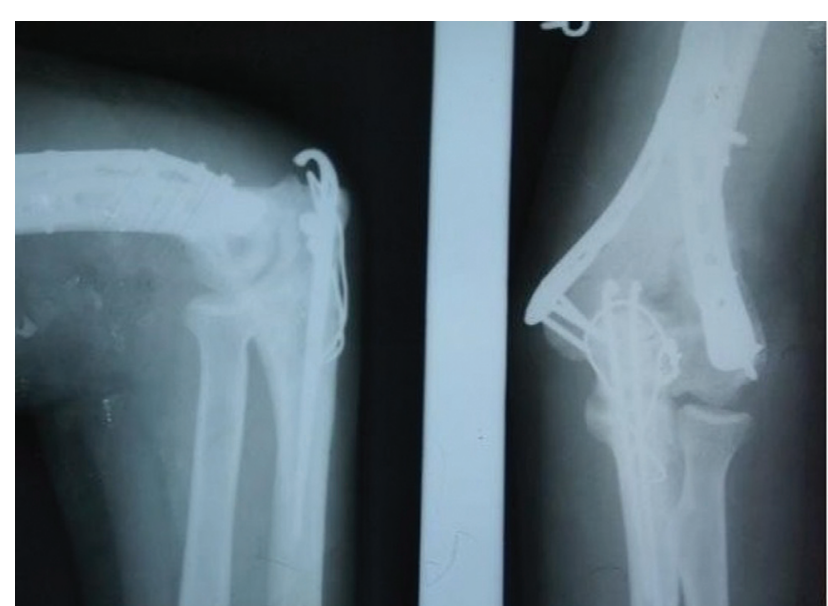

Fig. 3 Abordagem transolecraniana do caso-1: raio X no último seguimento.
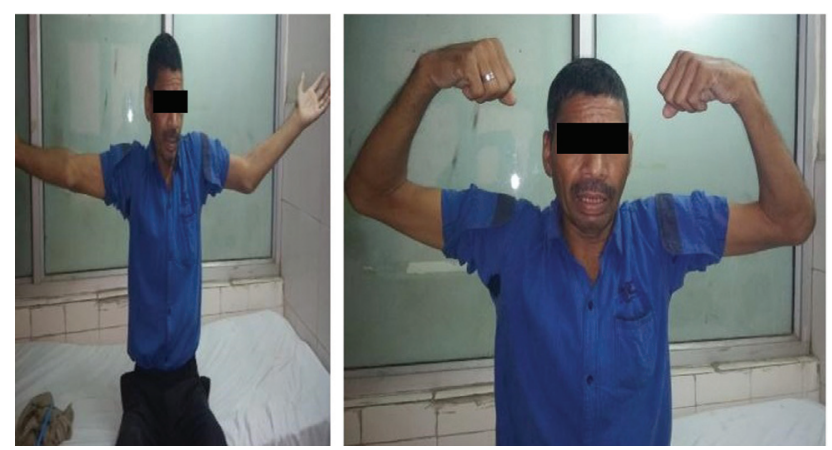

Fig. 4 Abordagem transolecraniana do caso-1: amplitude de movimento no último seguimento.

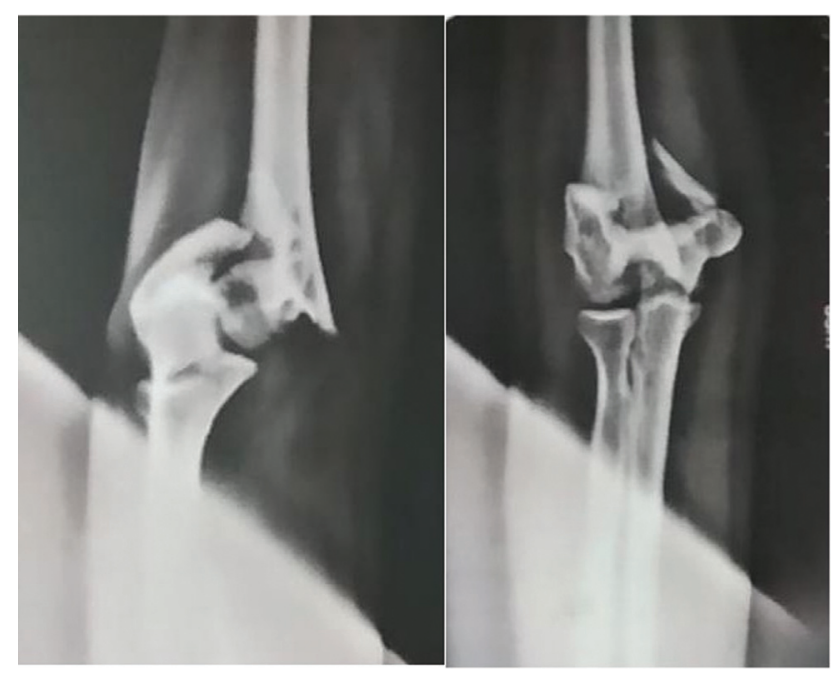

Fig. 5 Abordagem por reflexão do tríceps do caso-2: raio X préoperatório.

\section{Abordagem de reflexão do tríceps}

Uma incisão em forma de $S$ desleixado de $15 \mathrm{~cm}$ de comprimento foi feita no aspecto posterior do cotovelo, começando a aproximadamente $7,5 \mathrm{~cm}$ a $10 \mathrm{~cm}$ acima da ponta do olécrano superomedialmente, e terminando cerca de $5 \mathrm{~cm}$
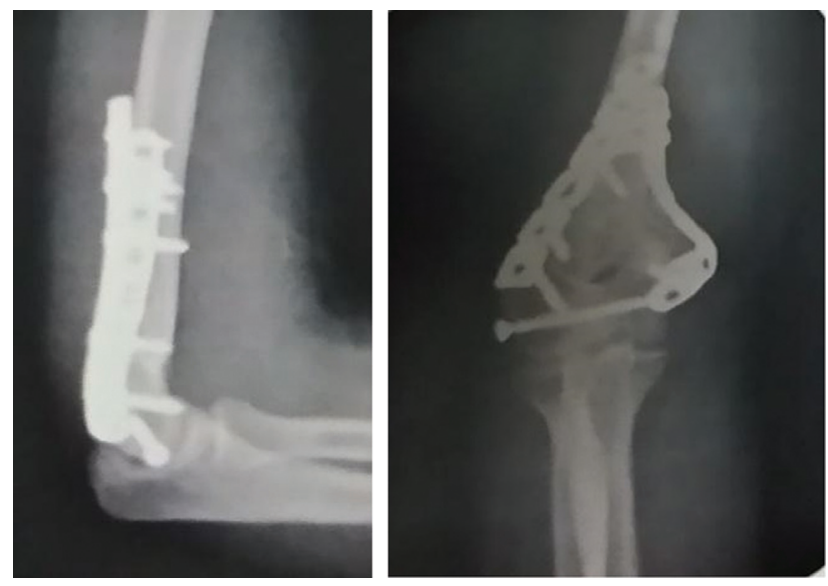

Fig. 6 Abordagem por reflexão do tríceps do caso-2: raio X no último seguimento.

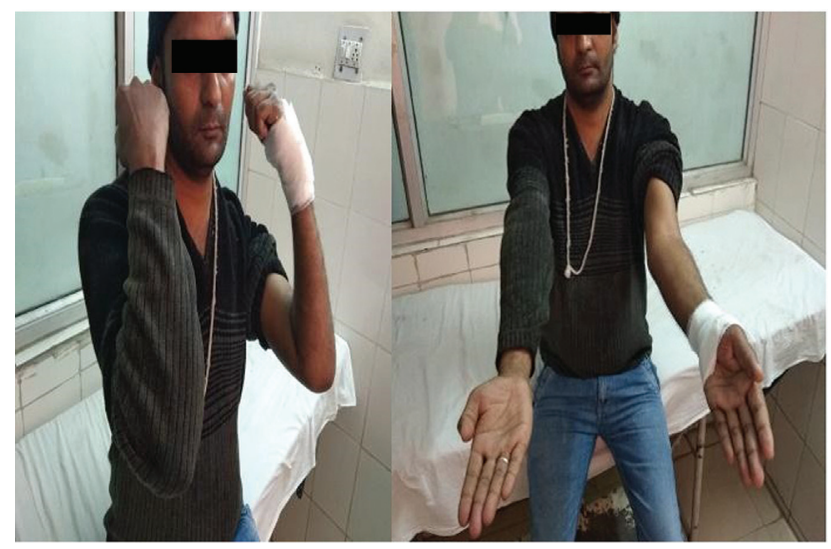

Fig. 7 Abordagem por reflexão do tríceps do caso-2: amplitude de movimento no último seguimento.

inferolateralmente para dissecar o nervo ulnar facilmente e evitar a necrose da pele. Após dissecar o nervo ulnar, uma separação foi feita entre o tríceps e o septo intermuscular medial, e o tríceps foi elevado ao aspecto posterior do úmero. $\mathrm{Na}$ lateral, o tríceps foi separado do septo intermuscular lateral e do úmero posterior junto com o músculo ancôneo. Ao levantar o tendão do tríceps, todo o úmero distal foi visualizado, e a fixação foi feita da mesma forma do que na osteotomia olecraniana (-Figuras 5-7).

\section{Manejo pós-operatório}

Os protocolos pós-operatórios foram os mesmos para ambas as abordagens. A inspeção foi feita no terceiro dia de pósoperatório, e, em seguida, o dreno foi removido. A extensão ativa da articulação do cotovelo foi permitida no terceiro dia de pós-operatório em ambos os procedimentos. Foram feitos raios X pós-operatórios do cotovelo operado (nas vistas anteroposterior e lateral). Os exercícios de flexão e extensão passiva do cotovelo foram iniciados no quinto dia de pósoperatório, e a mobilização livre foi permitida. Os pontos foram removidos no décimo segundo dia de pós-operatório, os pacientes receberam alta com instruções, e foram 
Tabela 1 Comparação entre os dois grupos de estudo

\begin{tabular}{|l|l|l|l|}
\hline & Grupo A & Grupo B & Valor de $\boldsymbol{p}$ \\
\hline Idade (anos) & $38,12 \pm 15,06$ & $34 \pm 14,11$ & $>0,05$ \\
\hline Perda de sangue (mL) & $222,78 \pm 34,93$ & $121,61 \pm 19,85$ & $<0,01$ \\
\hline Tempo de operação (minutos) & $92,67 \pm 8,73$ & $78,63 \pm 7,07$ & $<0,01$ \\
\hline Amplitude de movimento (graus) & $91,04 \pm 13,55$ & $92,65 \pm 19,07$ & $>0,05$ \\
\hline Pontuação no Mayo Elbow Performance Score & $82,91 \pm 11,60$ & $86,38 \pm 10,45$ & $>0,05$ \\
\hline $\begin{array}{l}\text { Pontuação no questionário Disabilities of the Arm, } \\
\text { Shoulder and Hand }\end{array}$ & $36,00 \pm 8,26$ & $34,57 \pm 9,50$ & $>0,05$ \\
\hline
\end{tabular}

chamados após uma semana. Posteriormente, os pacientes foram acompanhados em intervalos mensais por três meses, e, depois, de três em três meses por doze meses. Durante o seguimento, os pacientes foram examinados para se verificar a dor, infecção tardia, deiscência da ferida, parestesia, estado de consolidação da fratura, e qualquer complicação tardia, como falha do implante, rigidez, e não consolidação. A ADM foi registrada e as pontuações no Disabilities of the Arm, Shoulder and Hand (DASH) e no Mayo Elbow Performance Score (MEPS) foram calculadas em conformidade.

\section{Análise estatística}

As informações sobre ambos os grupos de pacientes foram coletadas e transferidas para uma planilha do programa Microsoft Excel (Microsoft Corp., Redmond, WA, EUA). Posteriormente, a análise dos dados foi feita na versão 17.0 do programa Statistical Package for the Social Sciences (SPSS Statistics for Windows, SPSS Inc., Chicago, IL, EUA). Foram aplicados testes estatísticos apropriados (o teste do quiquadrado foi utilizado para os dados categóricos, e o teste $t$ de Student e o teste de Mann Whitney foram utilizados para os dados não categóricos). Valores de $p \leq 0,05$ foram considerados estatisticamente significativos.

\section{Resultados}

No total, foram operados 58 pacientes, dos quais 8 foram perdidos durante o seguimento. Portanto, 50 pacientes (33 [66\%] homens e 17 [34\%] mulheres) com idades variando de 20 a 70 anos foram incluídos neste estudo. A maioria dos pacientes $(33 ; 66 \%)$ era de faixa etária fisicamente ativa, e houve predominância de casos de fratura fechada (46 pacientes; $92 \%$ ), com apenas 4 casos (8\%) de fratura aberta. A causa mais comum de lesão foi acidente de trânsito (AT), com $57,57 \%$, e o maior número de fraturas foi de tipo C2 da AO/OTA ( $\mathrm{n}=21 ; 42 \%)$ (-Tabela 1 ).

Tabela 2 Desfechos segundo o Mayo Elbow Performance Score

\begin{tabular}{|l|l|l|l|l|}
\hline & $\begin{array}{l}\text { Excelente } \\
(\geq \mathbf{9 0 )}\end{array}$ & $\begin{array}{l}\text { Bom } \\
(\mathbf{7 5 - 8 9 )}\end{array}$ & $\begin{array}{l}\text { Razoável } \\
(\mathbf{6 0 - 7 5 )}\end{array}$ & $\begin{array}{l}\text { Ruim } \\
(<\mathbf{6 0 )}\end{array}$ \\
\hline Grupo A & $24(80 \%)$ & $5(16 \%)$ & $1(4 \%)$ & 0 \\
\hline Grupo B & $15(75 \%)$ & $5(25 \%)$ & 0 & 0 \\
\hline
\end{tabular}

Tabela 3 Complicações observadas na amostra

\begin{tabular}{|l|l|l|}
\hline & Grupo A & Grupo B \\
\hline Infecção do tecido mole & 8 & 1 \\
\hline Neurapraxia do nervo ulnar & 2 & 0 \\
\hline Ossificação heterotópica & 0 & 1 \\
\hline Proeminência do implante & 1 & 0 \\
\hline $\begin{array}{l}\text { Consolidação atrasada do } \\
\text { processo olecraniano }\end{array}$ & 0 & 0 \\
\hline Neurapraxia do nervo radial & 0 & 0 \\
\hline
\end{tabular}

No grupo B, o tempo médio de operação foi de 78,63 minutos, e nogrupo A, de 92,62 minutos, e foi estatisticamente significativo $(p<0,01)$. A perda de sangue média no grupo B foi de $121,61 \mathrm{~mL}$, e no grupo A, de $222,78 \mathrm{~mL}$, e também foi estatisticamente significativa $(p<0,01)$. Na avaliação do desfecho de acordo com o MEPS, o grupo $B$ foi ligeiramente melhor em relação ao grupo $A$, mas não houve diferença estatisticamente significativa (-Tabela 2). Na avaliação do desfecho de acordo com o DASH o grupo A foi ligeiramente melhor em comparação com o grupo $B$, mas tampouco houve diferença estatisticamente significativa. A taxa de complicações foi mais elevada no grupo A do que no B.

\section{Complicação}

Infecção foi a complicação mais comum (9 casos) observada neste estudo, principalmente no grupo $A$, seguida de neurapraxia do nervo ulnar (2 casos), ossificação heterotópica (1 caso), e proeminência do implante (1 caso) (-Tabela 3 ).

\section{Discussão}

O desfecho funcional da fratura do úmero distal pode variar devido ao padrão variável das fraturas. Devido à baixa incidência desse tipo de fraturas, apenas algumas séries, mas com um número considerável de casos, foram publicadas. Sendo a maioria das fraturas intra-articulares, geralmente aceita-se que o tratamento por redução aberta e a fixação interna (RAFI) é o padrão, e seus objetivos são: a cicatrização do tecido mole sem infecção, a restauração da reserva óssea metafiseal, e a obtenção de uma articulação estável, indolor e móvel. ${ }^{5}$

O desfecho funcional ruim no longo prazo é mais comumente associado à diminuição da ADM devido à rigidez da 
imobilização prolongada. Portanto, a chave é a fixação estável para permitir movimentos precoces da articulação do cotovelo no pós-operatório. ${ }^{6}$ Foram descritas várias abordagens cirúrgicas a para fixação de fraturas distais do úmero: osteotomia olecraniana, abordagem por reflexão do tríceps com pedículo no ancôneo (RTPA), divisão de tríceps, ou as abordagens por reflexão do tríceps ou paratricipitais. ${ }^{7}$ A qualidade das evidências na literatura é de nível III ou IV. A experiência relatada com o uso da abordagem por reflexão do tríceps para tratar as fraturas distias do úmero em pacientes adultos é pequena. Pelo que sabemos, poucos estudos $^{8,9}$ compararam os resultados funcionais dessa abordagem com os da osteotomia olecraniana para o manejo por RAFI de fraturas distais do úmero. Por isso, este estudo foi realizado para comparar a osteotomia olecraniana e a reflexão do tríceps em termos da exposição ideal e do desfecho funcional.

Este estudo incluiu 50 pacientes com fraturas dos tipos $\mathrm{A}$, $B$ e $C$ da AO/OTA do úmero distal: 30 foram tratados por osteotomia olecraniana (grupo A), e 20 , por reflexão do tríceps (grupo B).

A média de idade da amostra foi de $43,03 \pm 12,05$ anos. A média de idade do grupo $A$ foi de $43,23 \pm 15,09$ anos, e do Grupo B, de 35,83 $\pm 14,01$ anos, e foram similares. A maioria dos pacientes era do sexo masculino: 33 (66\%). Também houve predominância masculina em outros estudos, como no de Bhandary et al. ${ }^{10} \mathrm{~A}$ maior incidência masculina pode refletir uma tendência dos homens a praticar mais atividades ao ar livre, o que os torna mais propensos a lesões.

Neste estudo, a incidência de fraturas abertas foi de $8 \%$ $(n=4)$, e todos esses pacientes foram submetidos a fixação definitiva em 1 semana, 3 por osteotomia olecraniana e 1 por reflexão do tríceps. A incidência de fraturas abertas foi comparável à de estudos anteriores feitos por Ek et al. ${ }^{11} \mathrm{e}$ Ali et al. ${ }^{12}$

Neste estudo, 21 (42\%) pacientes tinham fratura de tipo C2, 13 (26\%), C1, e 10 (20\%), C3. Os outros 6 (12\%) casos eram dos tipos A e B. Neste estudo, foram operados 5 pacientes com tipo C1, 9 pacientes com tipo C2, e 7 com fraturas tipo C3 no grupos $\mathrm{A}$, e 8 pacientes com tipo $\mathrm{C} 1,12$ com tipo $\mathrm{C} 2$, e 3 com tipo C3 no Grupo B. Ek et al. ${ }^{11}$ relataram 5 de 9 (55,55\%) casos de tipo C2 de AO/OTA. Ali et al. ${ }^{12}$ e Zhang et al., ${ }^{13}$ também relataram alta incidência de fraturas de tipo C2 AO/OTA de úmero distal, ou seja, 11 de 22 (50\%) e 25 de 67 (37,3\%), respectivamente.

Em nosso estudo, observamos que a abordagem por reflexão do tríceps é rápida, fácil de realizar (se o cirurgião for experiente), e possibilita uma boa redução nas fraturas com grandes fragmentos (tipos A, B, C1 e C2 de AO/OTA); e a abordagem de osteotomia olecraniana é melhor para a fixação das fraturas de tipo C3 (proporciona melhor visibilidade da superfície articular). A duração da cirurgia está diretamente relacionada ao tipo de fratura. Wilkinson e Stanley ${ }^{14}$ demonstraram que a diferença de visualização entre a reflexão do tríceps e a osteotomia olecraniana é a falta de visualização de $11 \%$ da superfíciena abordagem por reflexão do tríceps, e que até mesmo a osteotomia olecraniana deixa $43 \%$ da superfície invisível. Também de acordo com Ek et al., ${ }^{11}$ a abordagem por reflexão do tríceps proporciona exposição adequada dos locais de fratura.

No grupo B, o tempo médio da operação $(92,62 \pm 8,73$ minutos) foi menor do que o do grupo A (78,63 $\pm 7,02$ minutos). A diferença de tempo operatório foi estatisticamente significativa. Resultados semelhantes também foram observados no estudo de Zhang et al.: ${ }^{13}$ no grupo de osteotomia olecraniana a duração média da operação foi de 113,89 minutos, e no grupo de reflexão de tríceps, 89,03 minutos, com diferença estatisticamente significativa.

A avaliação dos desfechos da amostra deste estudo foi feita por meio de dois sistemas de pontuação: o MEPS, que é preenchido pelo médico com base em medição clínica e funcional, e o DASH, que é um questionário preenchido pelo paciente que avalia os componentes subjetivos da condição. No momento, não há controle ou valores normais para as pontuações no DASH. A pontuação média no DASH neste estudo foi de 35,25. A pontuação média no DASH do grupo A foi de 36,00, e do grupo B, 34,51; a pontuação média no DASH foi de 17,9 pontos no estudo feito por Ek et al. ${ }^{11}$ A média no MEPS da amostra deste estudo foi de 84,64 (variação: 75 a 100). No grupo A, a média no MEPS foi de 82,91, e no grupo B, 86,31. Neste estudo, segundo o MEPS, os desfechos foram classificados como excelentes em 24 (80\%) pacientes, bons em 5 (16\%), e razoáveis em 1 (4\%) paciente no grupo $A$, enquanto, no grupo $B$, excelentes desfechos foram observedos em 15 (75\%) pacientes, e bons desfechos em 5 (25\%). Nenhum resultado ruim foi obtido em nenhum dos grupos. Este achado é comparável ao do estudo feito por Zhang et al., ${ }^{13}$ cujas médias no MEPS foram de 85,56 e 87,71 para osteotomia olecraniana e reflexão de tríceps, respectivamente.

A média da ADM do cotovelo neste estudo variou de $12,81^{\circ}$ de extensão a $104,7^{\circ}$ de flexão (variação: $90^{\circ}$ a $130^{\circ}$ ). No seguimento final, a flexão média foi de $104,79^{\circ}$ (variação: $30^{\circ}$ a $140^{\circ}$ ), com extensão média de $12,81^{\circ}$ (variação: $0^{\circ}$ a $18^{\circ}$ ). A flexão média no grupo $A$ foi de $104,16^{\circ} \pm 9,16^{\circ}$, e, no $B$, $105,42^{\circ} \pm 12,99^{\circ}$. A extensão média no grupo $A$ foi de $12,87^{\circ} \pm 2,83^{\circ}$, e, no $B, 12,76^{\circ} \pm 5,63^{\circ}$. Assim, a ADM média foi de $91,84^{\circ}$, resultado similar ao de outros estudos. Ek et al. ${ }^{11}$ e Fernandez-Valencia et al. ${ }^{15}$ relataram ADMs médias de $90^{\circ} \mathrm{e}$ $112^{\circ}$, respectivamente.

A perda de sangue no grupo $B$ foi de $121,61 \pm 19,85 \mathrm{~mL}$, e, no grupo A, foi menor, de $222,78 \pm 34,93 \mathrm{ml}$, com diferença estatisticamente significativa $(p=0,01)$, similar aos resultados do estudo de Zhang et al. ${ }^{13}$

A infecção do tecidos moles foi a complicação mais comum (8 casos) encontrada neste estudo, principalmente nos pacientes do grupo A, seguida da neurapraxia do nervo ulnar (2 casos), da ossificação heterotópica (1 caso), e da proeminência do implante (1 caso),resultados similares aos do estudo feito por Chen et al. ${ }^{16}$ Nenhum caso de não consolidação ou de consolidação tardia foi observado neste estudo, talvez porque a banda de tensão foi usada como fixação final para a osteotomia olecraniana em vez de parafusos de $6,5 / 7 \mathrm{~mm}$ parcialmente enroscados com fio AI ou placa e parafusos.

Iselin et al. ${ }^{17}$ também concluíram que a abordagem por reflexão do tríceps é uma opção valiosa para RAFI em fraturas de intra-articulares do úmero distal, com preservação da 
anatomia articular normal do olécrano e evasão das possíveis complicações associadas à osteotomia olecraniana.

\section{Conclusão}

A reflexão do tríceps resulta em menor tempo de operação, menor perda de sangue, e baixa taxa de complicações. Por outro lado, a osteotomia olecraniana proporciona melhor precisão da redução articular. Não houve diferenças significativas entre os dois grupos em se tratando de desfecho funcional. Por isso, propomos uma nova classificação, que é uma modificação da classificação AO/OTA, a fim de escolher uma abordagem posterior adequada para fratura distal do úmero. A nova classificação compreende dois tipos: o tipo 1 inclui os tipos 13A, 13B1, 13B2, 13C1 e 13C2 de OA/OTA, e o tipo 2 inclui o tipo 13C3 de OA/OTA. Para fraturas de tipo 1, a abordagem por reflexão do tríceps pode ser considerada, e para as de tipo 2, a osteotomia olecraniana.

\section{Suporte Financeiro}

Este estudo não contou com apoio financeiro. Todos os custos de coleta, análise, interpretação dos resultados e redação foram pagos exclusivamente pelos autores.

Conflito de Interesses

Os autores não têm conflito de interesses a declarar.

\section{Referências}

1 Court-Brown C, Heckman JD, McKee M, editors. Rockwood and Green's Fractures in Adults. 8th ed. Philadelphia: Wolters Kluwer Health; 2015

2 Rose SH, Melton LJ 3rd, Morrey BF, Ilstrup DM, Riggs BL. Epidemiologic features of humeral fractures. Clin Orthop Relat Res 1982;(168):24-30

3 Ring D, Jupiter JB, Gulotta L. Articular fractures of the distal part of the humerus. J Bone Joint Surg Am 2003;85(02):232-238

4 Singh H, Kanodia N, Singh R. Paratricipital two window approach for complex intraarticular distal humerus fractures: A prospective analysis of 27 patients. Chin J Traumatol 2019;22(06): 356-360
5 MacAusland WR. Ankylosis of the elbow: With report of four cases treated by arthroplasty. JAMA 1915;64:312-318

6 Alonso-Llames M. Bilaterotricipital approach to the elbow. Its application in the osteosynthesis of supracondylar fractures of the humerus in children. Acta Orthop Scand 1972;43(06):479-490

7 Muller ME, Allgower M, Willenegger H, Schatzker J, Bandi W. Manual of Internal Fixation: Technique Recommended by the AOGroup. New York: Springer-Verlag; 1970

8 Jupiter JB, Mehne DK. Fractures of the distal humerus. Orthopedics 1992;15(07):825-833

9 Morrey BF. Limited and extensile triceps reflecting exposures of the elbow. In: Morrey BF, editor. Master Techniques in Orthopaedic Surgery: The Elbow. New York: Raven Press; 1994:3-20

10 Bhandary B, Shetty S, Kassim MS, Moha A. Open reduction internal fixation with triceps-sparing approach in distal humerus fracture: an experience from a tertiary level hospital in Mangalore. Int J Res Orthop 2019;5(02):345-349

11 Ek ET, Goldwasser M, Bonomo AL. Functional outcome of complex intercondylar fractures of the distal humerus treated through a triceps-sparing approach. J Shoulder Elbow Surg 2008;17(03): 441-446

12 Ali AM, Hassanin EY, El-Ganainy AE, Abd-Elmola T. Management of intercondylar fractures of the humerus using the extensor mechanism-sparing paratricipital posterior approach. Acta Orthop Belg 2008;74(06):747-752

13 Zhang C, Zhong B, Luo CF. Comparing approaches to expose type C fractures of the distal humerus for ORIF in elderly patients: six years clinical experience with both the triceps-sparing approach and olecranon osteotomy. Arch Orthop Trauma Surg 2014;134 (06):803-811

14 Wilkinson JM, Stanley D. Posterior surgical approaches to the elbow: a comparative anatomic study. J Shoulder Elbow Surg 2001;10(04):380-382

15 Fernández-Valencia JA, Muñoz-Mahamud E, Ballesteros JR, Prat S. Treatment of AO Type C Fractures of the Distal Part of the Humerus through the Bryan-Morrey Triceps-Sparing Approach. ISRN Orthop 2013;2013:525326

16 Chen G, Liao Q Luo W, Li K, Zhao Y, Zhong D. Triceps-sparing versus olecranon osteotomy for ORIF: analysis of 67 cases of intercondylar fractures of the distal humerus. Injury 2011;42 (04):366-370

17 Iselin LD, Mett T, Babst R, Jakob M, Rikli D. The triceps reflecting approach (Bryan-Morrey) for distal humerus fracture osteosynthesis. BMC Musculoskelet Disord 2014;15:406 